\title{
PROFESSIONALISM
}

ISSN: 1893-1049

Volume 11, No 2 (2021), e3711

https://doi.org/10.7577/pp. 3711

\section{Quandaries of Autonomy and Empowerment in Evidence-Based Nursing Care}

\author{
Marita Nordhaug \\ Oslo Metropolitan University, Norway
}

Contact: Marita Nordhaug, Oslo Metropolitan University, Norway, marita.nordhaug@oslomet.no

\section{Abstract}

Empowerment and evidence-based practice represent two influential principles in nursing care: that decision-making should be based upon the patient's autonomous choice, and the most up-to-date research findings, respectively. In this article, patient empowerment is taken to imply a transfer of control and power from the nurse to the patient through communication and care and acknowledging the patient's perspectives and values. Empowerment-based nursing may thus be central to enhancing a patient's autonomy. Evidence-based nursing combines up-to-date research findings, the nurse's clinical expertise and the patient's preferences. This article concerns some of the potential conflicts these principles may give rise to in everyday deliberations in nursing care. It is argued that patient empowerment and autonomy potentially both have paternalistic connotations. It is also questioned whether an increased emphasis on patient empowerment and autonomy may lead to a risk of diminished professional autonomy.

\section{Keywords}

Empowerment, evidence-based practice, nursing care, professional autonomy, patient autonomy 
Joe suffers from diabetes and due to increased self-care capacity, she receives nursing care in her home once a day. She has also developed a diabetic foot ulcer due to poorly regulated diabetes. In order to prevent further complications and slow wound progression, glycaemic control through lifestyle changes and wound care procedures are essential. Joe says she does not want to make any lifestyle changes. She is furthermore in doubt about the wound care procedures advised by the nurse. Instead, she wants her wound to be dressed with a particular aloe vera gel, which is a folk remedy. Research data on the effect and side-effects of the remedy are scarce. Bound by a professional duty to respect the patient's autonomy and prevent harm, as well as practise in accordance with principles of empowerment and evidence-based practice, what should the nurse do?

Chappel writes: "Ethics is the use of reason to answer the world-shaping question "how should life be lived?"' (2009, p. 3). When someone is in need and dependent on nursing care, whose use of reason, and what kinds of reasons, should be given weight in cases of conflicting views and concerns?

Empowerment and evidence-based practice represent two influential principles in nursing care: that decision-making should be based upon the patient's autonomous choice, and the most up-to-date research findings, respectively. In order to be evidence-based, nursing care combines up-to-date research findings, clinical expertise and experience, and the patient's preferences and desires in the situation at hand. This implies that the patient's articulated needs, preferences and desires form a knowledge base that should be taken into account, which is also a core element of empowerment thinking. Hence, respecting a patient's right to participate in daily decisions concerning his or her own health is of great importance in nursing care. Additionally, empowerment implies a transfer of control and power from the health care professional to the patient through communication and care, and for instance through interventions such as shared-decision making, guidance, patient education and supervision. Empowerment may thus be central to enhancing a patient's autonomy. At the same time, a hallmark of professionalism is professional autonomy. Professional autonomy is a precondition for professionals pursuing a certain collective good that members of other professions or people in general do not pursue (Nordhaug, 2017). In nursing care, the collective good pursued can be said to be adequate and individualised nursing care (Nordhaug, 2017). This takes place in nurse-patient relationships and include any action that nurses perform in order to promote and maintain the patient's well-being and prevent harm and suffering. According to Miller, professional autonomy is exercised when (a) decisions and actions in question are "his or her call", meaning he or she is the one to make the decision, and (b) the decision cannot be overridden by a superior (Miller, 2010). Notably, nursing care is conducted within health care organisations, through health care political guidelines, and very often in cooperation with other health care professions, which may affect the power balance between staff and thereby the ability to carry out professional autonomy in Miller's sense of the concept. Nursing care does not exist in a vacuum but 
takes place within systems of cooperation where different parties, such as leaders and physicians, depend upon each other to be able to carry out their mandates, but also where there may be power differences. Due to their complexity as well as the scope of this article, these kinds of power issues will not be further addressed here.

However, as we shall see in the next chapter, a central value in nursing care when it is based on the principles of empowerment, is that the patient is an expert on his or her situation. With a greater emphasis on patient's right, both ethically and legally, to autonomy and to participate in decisions concerning his or her health situation, nurses should also pay due attention to the patient's perspective. I believe it is fair to say that a key aspect of nursing ethics is that infringement of patient autonomy for reasons such as paternalistic interventions for the nurse's own professional autonomy, are unacceptable unless they prevent greater harm. But by enhancing the patient's autonomy and navigating the best upto-date research findings, do nurses run the risk of diminished professional autonomy, and if so, what are the implications for their responsibility regarding the principle "primum non nocere"? Or is the asymmetry in power in favour of the nurse reinforced by the considerable emphasis on factors such as the best up-to-date research findings in decisionmaking processes? These questions are underlying quandaries in this article. The article has the following structure: The first part highlights some ethical issues involved in empowerment thinking in nursing care. The second part briefly outlines some ethical implications of evidence-based nursing care. In the final part I provide an analysis of 12 cases of epistemological and ethical conflicts involved in evidence-based nursing, some of them rather trivial, some more complex ones.

This article does not concern acute or complex ethical dilemmas, such as life-or-death situations, but explores some of the potential conflicts that the principles of empowerment and evidence-based practice may lead to in everyday nursing care. It is important to note that the overall aim of this article is not to make normative claims or conclusions, but rather to map a terrain as a ground for further analysis and discussions concerning the complexities of two influential principles in nursing care. The reader may therefore find the analyses of some complex normative problems and epistemological conflicts simplistic.

\section{Empowerment, autonomy, and the patient's perspective in nursing care}

WHO's (1986) first international conference on health promotion, which set the scene for the Ottawa Charter of Health Promotion in 1986, was a breakthrough for empowerment thinking in health care (Tveiten \& Boge, 2014). The resulting emphasis on empowerment was much in line with the paradigmatic trend from paternalism to the prominence of patient autonomy and freedom of choice in health care. An anti-paternalistic account would for instance, as Scoccia (2013) writes, allege that "interference with the choices of wellinformed, competent adults cannot benefit because each is the best judge of where his self- 
interest lies" (Scoccia, 2013, p. 74). There are at least two central values underlying the concept of empowerment in health care. Firstly, we should acknowledge the idea that a patient is, usually, an expert on his or her situation. This implies recognising and respecting, at least to some extent, the patient's comprehension of needs, desires, aims and values. Secondly, control and power should be shared and transferred from the health care professional to the patient, through dialogue and interaction, and strategies such as shareddecision making. In nurse-patient relationships, an empowerment process implies a transfer of control and power from the nurse to the patient through health pedagogical strategies such as guidance, teaching and shared-decision making, involving information-sharing, as well as emphasis on and concern for the patient's own experiences, desires and needs in everyday nursing care. Notably, the sharing and transfer of power is not dependent on specific health pedagogical strategies and interventions, but could, and should, also be an implicit part of nursing care that takes the patient's perspective into account.

Empowerment can be viewed both as a process and as an outcome (Gibson, 1991; Ryles, 1999; Tveiten \& Boge, 2014). In health care, empowerment is generally viewed as a process or strategy for achieving control over factors and decisions affecting one's health (Gibson, 1991), and of enhancing patients' autonomy and capacity to make an informed decision (Kapp, 1989). "The chief legal mechanism of empowerment in the health area", says Kapp (1989), "is the doctrine of informed consent" (p. 5). For the discussion that follows, WHO's definition of empowerment is an appropriate point of departure: "Empowerment is the process of increasing the capacity of individuals or groups to make choices and to transform those choices into desired actions and outcomes" (WHO, 2006, p. 17).

Despite the intuitively positive associations with the concept of empowerment, several ethical issues have yet to be properly addressed in the literature. A recent thematic synthesis of concept analyses of empowerment with regard to health care users' perspectives, revealed that the user perspective and involvement in empowerment entailed challenges of equality in health care relationships. Furthermore, questions of power were scarcely discussed in the included articles (Halvorsen et.al., 2020). There has also been little investigation of whether the use of empowerment strategies to enhance patients' capacity to make informed decisions may in some cases threaten, rather than improve, patient autonomy. In some sense, as discussed by Halvorsen et.al. (2020) and Tengland (2016), empowerment processes may be assumed to have paternalistic undertones, tending to be more like an approach to changing behaviour.

Underlying the prima facie principles of empowerment is the value placed on respecting patient autonomy in health care. According to Beauchamp and Childress (2007), personal autonomy encompasses self-rule that is "free from both controlling interferences by others and from certain limitations such as an inadequate understanding that prevents meaningful choice (2007, p. 99). Autonomy is also frequently articulated by stating that an action is autonomous if it is performed intentionally, with understanding and without controlling 
influences (Faden and Beauchamp 1986). Respecting autonomy also implies accepting that some people do not want to take control over their own lives, or to participate in empowerment processes aimed at enhancing their consent, and we should respect the fully informed patient who still does not want to consent, but instead wishes to be dependent on health professionals (Kapp, 1989).

As Kapp (1989) correctly points out, decision-making power must be accepted voluntarily. This means that neither empowerment nor autonomy can be forced upon someone. A central question is then: is the outcome of an empowerment process aimed at increasing autonomy and capacity to consent, compatible with an accepted account of autonomy and consent, or does participation in this empowerment process presuppose autonomy and capacity to consent in the first place? If the former is true without the latter being true, empowerment may have some paternalistic undertones. Obviously, no one can be forced to be empowered. The concepts of autonomy and consent are usually discussed in relation to medical treatment or participation in research, and not as much in relation to (basic) nursing care. For instance, a patient who legitimately refuses to receive life-extending medical treatment might nevertheless be in need of nursing care interventions requiring consent, such as aiding their self-care regarding an adequate nutrition plan in order to reduce painful symptoms.

This is important, since the initiation of empowerment in health care seems to be directed towards people in particularly vulnerable situations. Furthermore, being in need of nursing care very often means being in a very vulnerable position. Indeed, an increasing number of patients in need of nursing care have dementia or other forms of cognitive impairments, which challenge their capacity of consent in the first place, as well as their capacity to participate in empowerment processes aimed at enhancing their consent. This is awkward as it suggests that participation in empowerment processes to enhance capacity to consent, in some sense, presupposes the possession of abilities required for such a capacity. In such situations, the exercise of autonomy is dependent on the existence of caring and trusting relationships (Lôhmus, 2015).

Finally, we return to WHO's definition of empowerment where the outcome of the empowerment process should be an increased capacity to make a choice, and an ability to transform that choice into desirable actions and outcomes. But what does "increasing" capacity amount to in a nursing care context? Is it a stronger sense of empowerment to make a choice or, alternatively, greater capacity (in some sense) to actually make own choices (Kieffer, 1983). Furthermore, what does it mean that the choice should be transformed into "desirable actions and outcomes"? Desirable according to whom? The nurse and the patient may have different opinions about what the desirable outcome of a situation should be. An empowerment process may, therefore, result in a choice and action that may conflict with what is professionally desirable and recommendable. However, at best, empowerment implies a transfer of control and power from the nurse to the patient 
through communication and care and should be central to maintaining or enhancing a patient's autonomy.

\section{Evidence-based nursing care}

Since the early 1990s, evidence-based practice (EBP) has also become very influential in many areas of health care such as nursing care. As stated previously, in order to be evidence-based, nursing care combines up-to-date research findings, clinical expertise and experience, and the patient's preferences and desires in the situation at hand. Evidencebased practice states that clinical decision-making should be based, as far as possible, on "the most up-to-date research findings" (Gupta, 2014, p. 1). EBP prioritises certain types of research data in decision-making processes, where randomized controlled trials take priority in the evidence hierarchy. In nursing care, this gives rise to at least two problems. Firstly, how much weight should be assigned to a patient's own preferences and values in situations where they conflict with "evidence of best effect"? Furthermore, should professionals set aside their experiences and professional values when they conflict with either evidence or the patient's values, or both? Secondly, the knowledge base, especially when it comes to randomized controlled studies on nursing care interventions, is scarce. I believe that one of the important reasons for this is that many nursing interventions are not equipped for randomized controlled studies, but instead focus on patients' experiences, which results in lower-level studies in the evidence hierarchy.

Interestingly, there is no evidence of the kind preferred by evidence-based medicine, whereby adherence to it will lead to improved health outcomes (Gupta, 2014). Gupta states that according to proponents of EBP (a term used interchangeably with "EBM"), "to practice anything but EBM would knowingly lead patients to less effective interventions and worse health" (Gupta, 2014, p. 2). As Gupta writes, the implicit ethical justification of evidencebased practice is therefore that "we should practice EBM because it is the best (most accurate) way to help patients achieve improved health" (2014, p. 2). This utilitarian justification is one way of comprehending the ethical foundation of evidence-based practice. Yet, as Gupta emphasises, there are other ethically relevant considerations in clinical decision-making apart from what is likely to lead to the kinds of health outcomes typically evaluated by clinical studies. As Gupta points out, in the early years, the EBP approach was criticised for being a cookbook approach that left no room for patient values and own preferences. Indeed, many sources of value are embedded in decision-making and EBP, but how they should be balanced in the event of conflict or doubt is still open to discussion and will be topic in the final part of this article.

\section{Evidence-based practice, empowerment, and autonomy}

Managing epistemological and ethical conflicts involved in evidence-based nursing care is replete with challenges. My aim in the following is not to lean on normative conclusions, but 
to briefly untangle, and to some extent discuss, 12 combinations of the three elements of evidence-based nursing. In the following, I use the term 'intervention' to represent any action nurses perform, or might perform, in order to promote and maintain the patient's well-being and prevent harm and suffering in a clinical situation.

First, consider these four scenarios:

1) There is no evidence of effect of intervention X. The patient does not want $X$. According to the nurse's professional experience, there is no effect or advantages of $X$.

2) There is no evidence of effect of intervention $X$. The patient does not want $X$. According to the nurse's professional experience, there is some effect and some important advantages of $\mathrm{X}$.

3) There is no evidence of effect of intervention $X$. The patient wants to have $X$. According to the nurse's professional experience, there is no effect or advantages of $X$.

4) There is no evidence of effect of intervention X. The patient wants to have $X$. According to the nurse's professional experience, there is some effect, and some important advantages of $\mathrm{X}$.

Notably, "no evidence of effect" only implies that no research has been conducted that can verify the effect of the intervention. Hence, the intervention might have a desirable effect, but this has, not (yet) been documented. It may also be the case that qualitative studies demonstrate that patients have valuable experiences concerning the intervention. However, according to the evidence hierarchy, this would not count as evidence of effect.

Subsequently, in any of the four cases above, the epistemological base evidence is unclear.

There is clearly no good reason to carry out the intervention in case 1 , since none of the three elements of evidence-based practice seem to support it. Indeed, such situations are not likely to occur very often in practical nursing care as it would imply that "We have something $(X)$, but we do not know if it works. What we do know is that the patient does not want $X$, and our professional experience does not support $X$ either. Should we then apply X or not?". Hence, case 1 appears to be a theoretical possibility of minor clinical relevance.

The epistemological conflict in case 2 and 4 probably fits with many patient situations in nursing care. Many nursing care interventions do not enter randomized controlled studies aimed at proving effects, but instead rely on professional experiences and values, as well as addressing the patients' own preferences. Indeed, nursing interventions need to be responsive to the complexities of the situation at hand, and besides, some nursing 
interventions are not subject to any studies at all. Case 4, then, should not necessarily be a source of conflicting concerns. Case 2 is not radically different from case 1 , but in case 2 the nurse's experience indicates that the intervention may benefit the patient, although there is no evidence supporting it. In these two cases, as in any of the other cases here presented, the question is of course to what extent there are more than one intervention available. It is also important that in clinical situations, there are numerous interactive components involved. Sensitivity to what is at stake for the patient in the situation is therefore of vital importance. A patient hesitating or even refusing an unstudied intervention aimed at reducing his or her harm and suffering may give rise to an ethical conflict between respecting a patient's autonomy, and the nurse's duty to prevent (further) harm or suffering. Noteworthy, carrying out an intervention against a patient's approval, even with the best intentions, may in itself induce harm in the patient.

In case 3, the patient wants an intervention that is not evidence-based, but clinical experience does not support the patient's preference. An example of a similar case is found in Gupta's (2014) study, where doctors were asked what they would do if a patient wanted long-term benzodiazepines to be able to fall asleep. Since, as Gupta writes, this is not the best way of facilitating good sleep, and the patient risks harmful side effects, doctors withheld the prescription citing their professional ethical duty to prevent harm. In Gupta's study, if the patient wanted something that a health care professional deemed unsuitable, the participants believed excluding that option was consistent with EBP. This was so even the case in the absence of harmful side effects (Gupta, 2014). There is a paternalist undertone in clinical decisions that do not accept patients' preferences. Consider Joe's situation in the example in the beginning of this article. Although there is scarce evidence of the effect (and advantages) of aloe vera gel on healing diabetic wounds, patients may have positive experiences concerning its effect. However, notably, a patient who prefers aloe vera gel on her diabetic wound instead of the wound dressing offered by the nurse, is in a state of needing a wound dressing, which is a need related to preventing or alleviating further harm and suffering. It is the categorical need for a wound dressing per se, not the desire for aloe vera gel per se, that obligates the nurse to act. For instance, it is not irrational to want to apply aloe vera gel to a diabetic wound, so, given the scarce research data on the effect of this intervention, is there any good reason not to accept the Joe's preference? Probably not, and why not? And through transfer of control, and acknowledging the patient as an expert on his or her situation, empowerment-based nursing care make the patient's case even stronger. This is interesting, because in situations similar to case 3, empowerment-based nursing care may imply supporting a preference which is not in accordance with professional suggestions or standards. That said, this does not imply that a nurse always should act according to a patient's desires or preferences when this conflicts with professional standards, or, say, experience.

Let us now consider four other scenarios: 
5) There is evidence of no effect of $X$. The patient does not want $X$. According to the nurse's professional experience, there is no effect or advantages of $X$.

6) There is evidence of no effect of $X$. The patient does not want X. According to the nurse's professional experience, there is some effect and some important advantages of $\mathrm{X}$.

7) There is evidence of no effect of $X$. The patient wants $X$. According to the nurse's professional experience, there is no effect or advantages of $X$.

8) There is evidence of no effect of $X$. The patient wants $X$. According to the nurse's professional experience, there is some effect and some important advantages of $X$.

It should go without saying that case 5 should be ruled out due to the lack of any good reason to perform it. Case 6 appears to be similar to case 2 above, except that there is evidence here of no effect. Now, in both case 6 and 7 we are faced with an interesting question: To what extent can an intervention which is proved to be ineffective, yet be ethically acceptable? First of all, if an option is known to be harmful, it should naturally be eliminated. Accommodating a patient's preference should not go against the professional duty to prevent harm. However, as long as the option is not harmful, though ineffective, and the patient wants it, it may be ethically legitimate to accept the patient's preference. This is particularly relevant when we follow the principles of empowerment to safeguard patient autonomy and prevent paternalism.

What then about case 8 ? The only morally relevant difference from case 4 is that in case 8 there is evidence of no effect. Hence, according to research, the intervention in question does not work. Carrying out such an intervention will thereby constitute a non-evidencebased form of practice. This does not, however, imply that it is an unethical practice as long as it is in line with the patient's wishes and the nurse's experience. The requirement to respect a patient's right to make decisions concerning his or her health conflicts with the ethical obligation to promote the patient's health or alleviate their suffering. Dilemmas may also arise, not because of a lack or weak evidence of the effect of an intervention, but because of conflicts between incommensurably different values, such as a patient's autonomy and the nurse's duty to prevent harm and alleviate suffering.

Let us now consider four cases, where there is a compelling epistemological base due to evidence of the effect of the intervention in question.

9) There is evidence of effect of X. The patient does not want X. According to the nurse's professional experience, there is no effect or advantages of $X$. 
10) There is evidence of effect of $X$. The patient does not want $X$. According to the nurse's professional experience, there is some effect and some important advantages of $X$.

11) There is evidence of effect of $X$. The patient wants $X$. According to the nurse's professional experience, there is no effect or advantages of $X$.

12) There is evidence of effect of $X$. The patient wants $X$. According to the nurse's professional experience, there is some effect and some important advantages of $X$.

The last example, case 12, represents the ideal and desired situation in evidence-based practice. In case 11, there is evidence of effect of the intervention, and it is in line with the patient's preference. Respecting a patient's autonomous choice to have an intervention which is proven effective is not controversial. Hence, neither case 11 or 12 are of interest here. But what about case 9 and 10 ?

At first sight, case 9 appears to be a peculiar example. This is a situation where professional experience indicates that the intervention in question has no advantage or desirable effect. However, research verifies the effect of the very same intervention, suggesting an epistemological incompatibility between two different knowledge bases. The fact that the patient does not want the intervention further complicates the picture. We should bear in mind here that randomized controlled trials take priority in the evidence hierarchy in decision-making processes. This hierarchy of evidence does not take all the complexities of a specific patient situation into account. This may help explain why professional experience does not correspond with what evidence tells us. It also emphasises the importance of not only paying attention to the higher levels of the evidence hierarchy, but also to the lower levels of the hierarchy where for instance descriptive single qualitative studies on experiences provide important knowledge in a decision-making process. Hence, it may very well be the case that although there is evidence of the effect of a certain intervention, qualitative studies, for instance, may show that patients have undesirable experiences that should be paid attention to. Insight into other patients' experiences and preferences provide the nurse with important knowledge in decision-making processes. Knowing what the best course of action is not only requires awareness of the different epistemological positions informing nursing practice, but also sensitivity to and awareness of the particularities in the situation at hand, as well as a commitment to professional ethical values such as preventing harm. Indeed, we expect nurses to perform to the best of their ability.

Whether to respect a patient's autonomous choice to not accept the intervention in question, which is the question in case 10 , naturally depends on the particularities of the situation at hand. For instance, when vital needs are at stake, and the patient's refusal will 
reduce the likelihood of survival, nurses (as well as doctors) are faced with an ethical dilemma between their professional duty to respect the patient's autonomy, and the principle of primum non nocere. However, this is not the type of situation I am interested in here. Unlike the above example, the daily conflict situations in nursing care are generally not of a life-or-death nature, but concern needs related to preventing harm and alleviating suffering, as well as needs related to increasing the patient's well-being. An example is a patient who refuses lifestyle changes although he or she is aware of the high risk of cardiac diseases. In such situations, increasing the patient's evidence-responsiveness with respect to her own decision-making process may enable the nurse to perform the duty to prevent harm by confronting the patient. However, educating and guiding the patient about the risks and benefits of the different choices to increase their compliance with evidence and nurses' professional advice incurs a risk of paternalism. Indeed, nurses should also pay attention to personal as well as cultural values underlying a patient's refusal or noncompliance when making use of empowerment strategies, such as dialogue and supervision, in order to increase patients' capacity to make desirable (with reference to the definition of empowerment above) decisions and actions. There is a very strong emphasis here on consideration of patients' own experiences, comprehension of needs, values and desires. Empowerment may thus be central to enhancing a patient's autonomy. But it is also possible that empowerment strategies, in cases of conflict between the professional's duty to prevent harm and promote well-being, and the patient's autonomy, also become a manipulative strategy. On the other hand, the empowerment strategies imply a transfer of control and power from the nurse to the patient. A question then arise whether a transfer of control and power also implies a transfer of responsibility, leaving the patient with a kind of burden, and the nurse with some form of decreased professional autonomy.

\section{Conclusions}

Nurses' moral obligation to care for patients in accordance with principles of empowerment, and evidence-based practice raises some difficult issues where there is conflict between different knowledge bases and values such as respecting a patient's autonomy, and the nurse's own professional experience and preferences. The article identifies two problematic issues concerning empowerment and autonomy. Firstly, empowerment strategies may be a manipulative and paternalistic intervention. Secondly, by enhancing a patient's autonomy, there is also a danger of diminished professional autonomy, especially in cases where the evidence-base is scarce or lacking. As emphasised in the beginning of this article, the overall aim of this article is not to make normative claims or conclusions, but to map a terrain as a ground for further analysis and discussions concerning the complexities of two influential principles in nursing care. 


\section{Acknowledgments}

This work was partially supported by the Research Council of Norway, project number 250503, "Autonomy and manipulation: Enhancing consent in the health care context".

\section{Article history}

Received: 29 Jan 2020

Accepted: 13 Jan 2021

Published: 06 Aug2021

\section{References}

Beauchamp, T.L, \& Childress, J.F. (2007). Principles of biomedical ethics (6.ed.). New York: Oxford University Press.

Chappell, T. (2009). Ethics and experience: Life beyond moral theory. Durham: Acumen. https://doi.org/10.1017/UPO9781844654161

Faden, R.R., \& Beauchamp, T.L. (1986). A history and theory of informed consent. Oxford: Oxford University Press.

Gibson, C. (1991). A concept analysis of empowerment. Journal of Advanced Nursing, 16(3), 354-361. https://doi.org/10.1111/j.1365-2648.1991.tb01660.x

Gupta, M. (2014). Is evidence-based psychiatry ethical? Oxford: Oxford University Press. https://doi.org/10.1093/med/9780199641116.001.0001

Halvorsen, K., Dihle, A., Hansen, C., Nordhaug, M., Jerpseth, H., Tveiten, S., Joranger, P., \& Knutsen, I. R. (2020). Empowerment in healthcare: A thematic synthesis and critical discussion of concept analyses of empowerment. Patient Education and Counseling, 103(7), 1263-1271. https://doi.org/10.1016/i.pec.2020.02.017

Kapp, M. (1989). Medical empowerment of the elderly. The Hastings Center Report, 19(4), 57. https://doi.org/10.2307/3562292

Kieffer, C. (1983). Citizen empowerment: A developmental perspective. Prevention in Human Services, 3(2-3), 9-36. https://doi.org/10.1300/J293v03n02 03

Lôhmus, K. (2015). Caring autonomy. Cambridge: Cambridge University Press. https://doi.org/10.1017/CBO9781139976688

Miller, S. (2010). The moral foundations of social institutions: A philosophical study. Cambridge: Cambridge University Press. https://doi.org/10.1017/CBO9780511818622

Nordhaug, M. (2017). Partiality and justice in nursing care. London: Routledge. https://doi.org/10.4324/9781315211381 
Ryles, S. (1999). A concept analysis of empowerment: its relationship to mental health nursing. Journal of Advanced Nursing, 29(3), 600-607. https://doi.org/10.1046/j.1365-2648.1999.00928.x

Scoccia, D. (2013). The right to autonomy and the justification of hard paternalism. In: C. Coons \& M. Weber (Eds.). Paternalism: Theory and practice, (74-92). Cambridge University Press. https://doi.org/10.1017/CBO9781139179003.005

Tengland, P. A. (2016). Behavior change or empowerment: On the ethics of health promotion goals. Health Care Anal, 24(2016), 24-46.

https://doi.org/10.1007/s10728-013-0265-0

Tveiten, S. \&Boge, K. (2014). Empowerment i helse, ledelse og pedagogikk: Nye perspektiver. [Empowerment in health care, leadership, and pedagogics: New perspectives]. Oslo: Gyldendal Akademiske. [In Norwegian]

World Health Organization (WHO). (1986). The Ottawa Charter for Health Promotion. Geneva: World Health Organization. Retrieved from: https://www.who.int/healthpromotion/conferences/previous/ottawa/en

World Health Organization (WHO). (2006). What is the evidence of effectiveness of empowerment to improve health? Retrieved from:

https://www.euro.who.int/en/data-and-evidence/evidence-informed-policymaking/publications/pre2009/what-is-the-evidence-on-effectiveness-ofempowerment-to-improve-health 